ITEP/TH-72/07

\title{
Hamiltonian Formalism in the Presence of Higher Derivatives
}

\author{
A.Morozov \\ ITEP, Moscow, Russia
}

\begin{abstract}
A short review of basic formulas from Hamiltonian formalism in classical mechanics in the case when Lagrangian contains $N$ time-derivatives of $n$ coordinate variables. For non-local models $N=\infty$.
\end{abstract}

\section{Introduction}

Theories with higher derivatives were long at the periphery of theoretical physics. The basic reason for this is that they do not appear in everyday physical problems. This in turn can have a reason: if, as many believe these days, observable world is described by a low-energy limit of some yet-unknown fundamental theory, then it is naturally governed by Lagrangian dynamics with lowest possible - this means a pair of first - time-derivatives and Newton law includes only acceleration. From this point of view there is no restriction on the number of derivatives in the fundamental theory, and higher derivative terms are indeed present in most approaches, from QFT formulations of string and M-theory [1] to pure QFT models like asymptotically safe gravity [2] or (the quantum version of) the recent $E_{8}$ unification model [3]. It goes without saying that various non-local and innumerable non-commutative models all fit into category of higher-derivative theories. More than that, even the ordinary physical theories, like classical electrodynamics, appear inconsistent without higher derivatives, if one includes radiation phenomena and allows space-time dimension to be greater than 4 - like one does, for example, in amusing $\mathrm{TeV}$-gravity models [4]. In these circumstances the resolution of radiation friction and electromagnetic mass "problems" requires inclusion into the bare ("fundamental") action of counter-terms which not only renormalize mass (as in $d=4$ ), but necessarily include higher derivatives [5]. Of course, higher derivatives are also used for purposes of UV regularization in more formal context, especially in gauge invariant and supersymmetric models [6], even if inclusion of such terms is not physically unavoidable. Last but not the least, higher-derivative terms are the common place in all effective theories, from solid state physics to quantum gravity.

For all these reasons the higher-derivative dynamics is slowly gaining new attention, see [7]-[18] for the relatively recent discussions from various viewpoints, as well as [19]-[34] for some classical papers and monographs. It should be emphasized that this almost-untouched ground is very attractive from the point of view of "theoretical theory" and is intimately related to modern topological theory [35], $L_{\infty}^{(n)}$ structures a la [36], non-linear algebra [37] etc. Since [38] it is known that when such theories are required to be reparametrization invariant (what is the case in most of thinkable applications) new phenomena of outstanding beauty occur. Of special interest is symplectic geometry behind such theories [39, 40].

This short note is devoted to the 0 -th chapter of higher-derivative theory. It contains a short list of elementary formulas - well-known to a narrow class of interested people ever since [19] - about classical Lagrangian and Hamiltonian dynamics, which can be used for comparison with results of various more-advanced approaches.

\section{Lagrangian formalism}

Consider the classical mechanics with the action

$$
S\left\{q^{\alpha}(t)\right\}=\int L d t
$$

where Lagrangian $L\left(q^{\alpha}, d_{t} q^{\alpha}, \ldots,\left(d_{t}^{N} q^{\alpha}\right)\right)$ depends on the first $N$ time-derivatives $q_{i}^{\alpha} \equiv d_{t}^{i} q^{\alpha}$ of $n$ coordinate variables $q^{\alpha}=q_{0}^{\alpha}, \alpha=1, \ldots, n$. Obviously,

$$
\dot{q}_{i}^{\alpha}=q_{i+1}^{\alpha}
$$


Introduce the variational derivatives w.r.t. $q_{i}^{\alpha}$ for all $i \geq 0$ :

$$
\delta_{\alpha}^{i-1} \equiv \partial_{\alpha}^{i}-d_{t} \partial_{\alpha}^{i+1}+d_{t}^{2} \partial_{\alpha}^{i+2}-\ldots,
$$

where $\partial_{\alpha}^{i}=\partial / \partial q_{i}^{\alpha}$ and the momenta

$$
\Pi_{\alpha}^{i}=\delta_{\alpha}^{i} L
$$

These operators are related by time-derivatives:

$$
\dot{\delta}_{\alpha}^{i-1} \equiv d_{t} \delta_{\alpha}^{i-1}=\partial_{\alpha}^{i}-\delta_{\alpha}^{i}
$$

in a way, dual to (2).

The Euler-Lagrange equations of motion are

$$
\Pi_{\alpha}^{-1} \equiv \delta_{\alpha}^{-1} L=0 .
$$

These are equations of order $2 N$ in time-derivatives. Initial conditions are imposed on $q^{\alpha}, d_{t} q^{\alpha}, \ldots,\left(d_{t}^{2 N-1} q^{\alpha}\right)$.

Note that superscripts are not powers. In what follows we suppress indices $\alpha$ in some formulas.

\section{Hamiltonian formalism}

The phase space is $2 N n$-dimensional with canonically conjugate coordinates $q_{i}^{\alpha}$ and $\Pi_{\alpha}^{i}$, where $i=0, \ldots, N-1$. The $q_{N}^{\alpha}$ are functions of these independent variables.

The closed symplectic 2 -form

$$
\Omega=d \theta=\sum_{i=0}^{N-1} d \Pi_{\alpha}^{i} \wedge d q_{i}^{\alpha},
$$

is conserved:

$$
\dot{\Omega}=d_{t} \Omega=0,
$$

while the time-derivative of the associated pre-symplectic 1-form

$$
\theta=\sum_{i=0}^{N-1} \Pi_{\alpha}^{i} d q_{i}^{\alpha}
$$

is exact:

$$
\dot{\theta}=d L
$$

Hamilton equations state that

$$
\begin{array}{r}
\dot{q}_{i}^{\alpha}=q_{i+1}^{\alpha}=\frac{\partial H}{\partial \Pi_{\alpha}^{i}}, \\
\dot{\Pi}_{\alpha}^{i}=-\Pi_{\alpha}^{i-1}-\frac{\partial L}{\partial q_{i}^{\alpha}}=-\frac{\partial H}{\partial q_{i}^{\alpha}}
\end{array}
$$

with the Hamiltonian

$$
H(\Pi, q)=\sum_{i=0}^{N-1} \Pi_{\alpha}^{i} q_{i+1}^{\alpha}-L
$$

and $q_{N}^{\alpha}$ expressed through canonical variables.

Consider $\bar{S}(\bar{q}, \overline{\bar{q}})$ - the action (1), evaluated on the classical trajectory with the boundary conditions $\bar{q}_{i}^{\alpha}=$ $q_{i}^{\alpha}(\bar{t})$ and $\overline{\bar{q}}_{i}^{\alpha}=q_{i}^{\alpha}(\overline{\bar{t}}), i=0, \ldots, N-1$. Then

$$
\begin{gathered}
\frac{\partial \bar{S}}{\partial \bar{q}_{i}^{\alpha}}=-\bar{\Pi}_{\alpha}^{i}, \\
\frac{\partial \bar{S}}{\partial \overline{\bar{q}}_{i}^{\alpha}}=\overline{\bar{\Pi}}_{\alpha}^{i}
\end{gathered}
$$

All these relations are obvious generalizations of those in the simplest case of $N=1$, see [41]. 


\section{Proofs}

The proofs of above relations are straightforward:

$$
\begin{array}{r}
d_{t}\left[\sum_{i=0} d q_{i} \wedge d \delta^{i}\right]=\sum_{i=0}\left(d q_{i+1} \wedge d \delta^{i}-d q_{i} \wedge d \delta^{i-1}+d q_{i} \wedge d \partial^{i}\right)= \\
=-d q_{0} \wedge d \delta_{-1}+\sum_{i=0} d q_{i} \wedge d \partial^{i}
\end{array}
$$

When acting on $L$, the first term vanishes on equations of motion, $\delta^{-1} L=0$, while the second term becomes

$$
\sum_{i=0} d q_{i} \wedge d\left(\partial_{i} L\right)=\sum_{i, j=0} \frac{\partial^{2} L}{\partial q_{i} \partial q_{j}} d q_{i} \wedge d q_{j}=0
$$

Similarly

$$
d_{t}\left[\sum_{i=0} d q_{i} \delta^{i}\right]=\sum_{i=0}\left(d q_{i+1} \delta^{i}-d q_{i} \delta^{i-1}+d q_{i} \partial^{i}\right)=-d q_{0} \delta^{-1}+d
$$

Again, when acting on $L$ the first term vanishes on equations on motion.

Hamiltonian derivatives in (9) are:

$$
\begin{array}{r}
\frac{\partial H}{\partial \Pi^{i}}=q_{i+1}+\left(\Pi^{N-1}-\frac{\partial L}{\partial q_{N}}\right) \frac{\partial q_{N}}{\partial \Pi^{i}}, \\
-\frac{\partial H}{\partial q_{i}}=-\Pi^{i-1}\left(1-\delta_{i, 0}\right)+\frac{\partial L}{\partial q_{i}}-\left(\Pi^{N-1}-\frac{\partial L}{\partial q_{N}}\right) \frac{\partial q_{N}}{\partial q_{i}}
\end{array}
$$

The terms in brackets at the r.h.s. vanish because $\Pi^{N-1}=\delta^{N-1} L=\partial^{N} L$.

Finally, the variation of action $S\{q(t)\}$ under the variation $\delta q(t)$ of its argument is equal to

$$
\delta S=\int \sum_{i=0}^{N} \delta x_{i} \partial^{i} L=\oint \delta x_{j} \delta^{j} L+\int \delta^{-1} L
$$

On classical trajectory the second term vanishes, while the boundary contributions in the first term gives rise to $(11)$.

\section{Towards cohomological formulation}

The key role in above calculations is played by the operator

$$
\hat{A}=\sum_{i=1}(-)^{i} \partial^{i-1} \otimes \partial^{-i}=\frac{1}{1 \otimes 1+\partial \otimes \partial^{-1}} 1 \otimes \partial^{-1}=\frac{1}{\partial^{-1} \otimes \partial+1 \otimes 1} \partial^{-1} \otimes 1
$$

It is a formal inverse of $\partial$, which acts on the product by Leibnitz rule:

$$
(\partial \otimes 1+1 \otimes \partial) \hat{A}=\frac{1}{1 \otimes 1+\partial \otimes \partial^{-1}}\left(\partial \otimes \partial^{-1}+1 \otimes 1\right)=1 \otimes 1
$$

Conceptually, for $\partial=d_{t}$

$$
\Omega=\hat{A}_{*} d q \wedge \frac{\delta L}{\delta q}
$$

Indeed, for

$$
\frac{\delta}{\delta q_{i}}=\frac{\partial}{\partial q_{i}}-\partial \frac{\partial}{\partial q_{i+1}}+\ldots
$$

we have

$$
\partial \delta_{i+1}=\partial_{i}-\delta_{i}
$$

or

$$
\frac{\delta}{\delta q_{i+1}}=-\partial_{*}^{-1} \frac{\delta}{\delta q_{i}}=\ldots=(-)^{i} \partial_{*}^{-i} \frac{\delta}{\delta q}
$$

where $\partial_{*}^{-1}$ properly takes care of the $\partial / \partial q_{i}$ which lies in "cohomology" of $\partial$.

A more careful treatment should take into account the difference between $\hat{A}_{*}$ and $\hat{A}$. It is this difference that makes the above $\Omega$ non-vanishing, despite $\delta L=0$. Only the application of time-derivative $\partial$ eliminates $\hat{A}_{*}$, but without $\partial$ there is no vanishing. 


\section{$6 \quad$ Non-local examples}

Hamiltonian formalism is immediately applicable to arbitrary functionals, including non-local.

For $q$-quadratic examples one can take

$$
S\{q(t)\}=\int q \frac{1}{1+M^{-2} \partial^{2}} q d t
$$

or

$$
S\{q(t)\}=\int q(t) q(t+\epsilon) d t=\int q(t) e^{\epsilon \partial} q(t) d t
$$

(solutions of Euler-Lagrange equations in this case are antiperiodic functions $q(t+2 \epsilon)=-q(t)$ ), or, in general,

$$
S\{q(t)\}=\frac{1}{2} \int\left(\sum_{n} a_{n}\left(\partial^{n} q\right)^{2}\right) d t
$$

with time-independent $a_{n}$ (not a necessary restriction, of course).

Then

$$
\Pi_{i}=\delta^{i} L=\sum_{j=0}(-)^{j} a_{i+j+1} \partial^{i+2 j+1} q
$$

and

$$
\Omega=\sum_{i=0} d q_{i} \wedge d \Pi_{i}=\sum_{i, j \geq 0}(-)^{j} a_{i+j+1} d q_{i} \wedge d q_{i+2 j+1}
$$

Nota that terms $d q_{i} \wedge d q_{i+2 j}$ do not appear in this expansion. Since coefficients $A_{i j}$ in the matrix

$$
\Omega=\sum_{i<j} A_{i j} d q_{i} \wedge d q_{j}
$$

are time independent, they are forced to be of the form $A_{i j}=(-)^{j} A_{i+j}$ (familiar from the theory of Toda chain $\tau$-functions, see [42]) by the conservation condition:

$$
\dot{A}_{i j}+A_{i-1, j}+A_{i, j-2}=0
$$

\section{Reparametrization-invariant actions}

Transformations $t \rightarrow u(t)=t+\epsilon(t)$ :

$$
\begin{array}{r}
q_{1}^{\alpha} \equiv \dot{q}^{\alpha} \rightarrow u q_{1}^{\alpha} \rightarrow q_{1}^{\alpha}+\epsilon q_{1}^{\alpha}, \\
q_{2}^{\alpha} \equiv \ddot{q}^{\alpha} \rightarrow u^{2} q_{2}^{\alpha}+u \dot{u} q_{1}^{\alpha} \rightarrow q_{2}^{\alpha}+2 \epsilon q_{2}^{\alpha}+\dot{\epsilon} q_{1}^{\alpha}, \\
q_{3}^{\alpha} \rightarrow u^{3} q_{3}^{\alpha}+3 u^{2} \dot{u} q_{2}^{\alpha}+u \dot{u}^{2} q_{1}^{\alpha}+u^{2} \ddot{u} q_{1}^{\alpha} \rightarrow q_{3}^{\alpha}+3 \epsilon q_{3}^{\alpha}+3 \dot{\epsilon} q_{2}^{\alpha}+\ddot{\epsilon} q_{1}^{\alpha},
\end{array}
$$

or

$$
q_{k}^{\alpha} \equiv \partial_{t}^{k} q^{\alpha} \rightarrow q_{k}^{\alpha}+\sum_{l=0}^{k-1} C_{k}^{k-l-1} q_{k-l}^{\alpha} \partial_{t}^{l} \epsilon
$$

Invariance of the action means that for any $\epsilon(t)$

$$
\begin{aligned}
& \epsilon L=\epsilon\left\{\dot{q}^{\alpha} \frac{\partial}{\partial \dot{q}^{\alpha}}+2 \ddot{q}^{\alpha} \frac{\partial}{\partial \ddot{q}^{\alpha}}+\right.\left.3 q_{3}^{\alpha} \frac{\partial}{\partial q_{3}^{\alpha}}+4 q_{4}^{\alpha} \frac{\partial}{\partial q_{4}^{\alpha}}+\ldots\right\} L+ \\
&+\dot{\epsilon}\left\{\dot{q}^{\alpha} \frac{\partial}{\partial \ddot{q}^{\alpha}}+3 \ddot{q}^{\alpha} \frac{\partial}{\partial q_{3}^{\alpha}}+6 q_{3}^{\alpha} \frac{\partial}{\partial q_{4}^{\alpha}}+\ldots\right\} L+ \\
&+\ddot{\epsilon}\left\{\dot{q}^{\alpha} \frac{\partial}{\partial q_{3}^{\alpha}}+4 \ddot{q}^{\alpha} \frac{\partial}{\partial q_{4}^{\alpha}}+\ldots\right\} L+ \\
&+\left(\partial_{t}^{3} \epsilon\right)\left\{\dot{q}^{\alpha} \frac{\partial}{\partial q_{4}^{\alpha}}+\ldots\right\} L+\ldots
\end{aligned}
$$


or

$$
\sum_{m=0}^{\infty} K_{m} \partial_{t}^{m} \epsilon=0 \quad \text { i.e. } \quad K_{m}=\sum_{k \geq 1}^{\infty} C_{m+k}^{k-1} q_{k}^{\alpha} \frac{\partial L}{\partial q_{k+m}^{\alpha}}-L \delta_{m, 0}=0
$$

where binomial coefficients $C_{m+k}^{k-1}=\frac{(m+k) !}{(k-1) !(m+1) !}$.

As a corollary, the ordinary Hamiltonian (10) vanishes identically:

$$
\begin{gathered}
H=\sum_{i=0}^{N-1} \Pi_{\alpha}^{i} q_{i+1}^{\alpha}-L=\sum_{k \geq 1} q_{k}^{\alpha}\left(\frac{\partial L}{\partial q_{k}^{\alpha}}-\frac{d}{d t} \frac{\partial L}{\partial q_{k+1}^{\alpha}}+\frac{d^{2}}{d t^{2}} \frac{\partial}{\partial q_{k+2}^{\alpha}}-\ldots\right)-L= \\
=K_{0}-\sum_{k \geq 1}\left((k-1) q_{k}^{\alpha} \frac{\partial L}{\partial q_{k}^{\alpha}}+q_{k}^{\alpha} \frac{\partial^{2}}{\partial q_{k+1}^{\alpha} \partial q_{l}^{\beta}} q_{l+1}^{\beta}-\ldots\right)= \\
=K_{0}-\frac{d K_{1}}{d t}+\sum_{k}\left(\frac{(k-1)(k-2)}{2} q_{k}^{\alpha} \frac{\partial L}{\partial q_{k}^{\alpha}}+\frac{(k-1)(k+2)}{2} q_{k}^{\alpha} \frac{\partial^{2} L}{\partial q_{k+1}^{\alpha} \partial q_{l}^{\beta}} q_{l+1}^{\beta}+\right. \\
\left.+q_{k}^{\alpha} \frac{\partial^{2} L}{\partial q_{k+2}^{\alpha} \partial q_{l}^{\beta}} q_{l+2}^{\beta}+q_{k}^{\alpha} \frac{\partial^{3} L}{\partial q_{k+2}^{\alpha} \partial q_{l}^{\beta} \partial q_{m}^{\gamma}} q_{l+1}^{\beta} q_{m+1}^{\gamma}-\ldots\right)= \\
=K_{0}-\frac{d K_{1}}{d t}+\frac{d^{2} K_{2}}{d t^{2}}-\frac{d^{3} K_{3}}{d t^{3}}+\ldots=0
\end{gathered}
$$

As in every gauge invariant theory the Hamilton equations involve the constraint $\Phi$ - generator of gauge transformation

$$
\begin{aligned}
\dot{q}^{\alpha} & =\frac{\partial \Phi}{\partial \Pi_{\alpha}}, \\
\dot{\Pi}_{\alpha} & =-\frac{\partial \Phi}{\partial q^{\alpha}}
\end{aligned}
$$

- instead of the naive Hamiltonian [25].

A special case with no dependence on $q_{0}^{\alpha}$ and $N=1$ was studied in [38].

\section{Example of $n=2$ and $N=2$}

According to (27) in this case $L$ is a function of $q_{1}, q_{2}, v_{1}=\dot{q}_{1}, v_{2}=\dot{q}_{2}$ and $z=v_{1} a_{2}-v_{2} a_{1}=v_{1} \dot{v}_{2}-v_{2} \dot{v}_{1}$ of definite homogeneity degree:

$$
\left.3 z \frac{\partial L}{\partial z}\right|_{v_{1,2}}+\left.v_{1} \frac{\partial L}{\partial v_{1}}\right|_{v_{2}, z}+\left.v_{2} \frac{\partial L}{\partial v_{2}}\right|_{v_{1}, z}=L
$$

The momenta are equal to:

$$
\begin{gathered}
\Pi_{1}^{0}=\left.\frac{\partial L}{\partial v_{1}}\right|_{v_{2}, a_{1,2}}-\left.\frac{d}{d t} \frac{\partial L}{\partial a_{1}}\right|_{v_{1,2}, a_{2}}=\left.\frac{\partial L}{\partial v_{1}}\right|_{v_{2}, z}+\left.a_{2} \frac{\partial L}{\partial z}\right|_{v_{1,2}}+\frac{d}{d t}\left(\left.v_{2} \frac{\partial L}{\partial z}\right|_{v_{1,2}}\right)= \\
=\left.\frac{\partial L}{\partial v_{1}}\right|_{v_{2}, z}+\left.2 a_{2} \frac{\partial L}{\partial z}\right|_{v_{1,2}}+\left.v_{2} \frac{d}{d t} \frac{\partial L}{\partial z}\right|_{v_{1,2}}=\frac{\partial L}{\partial v_{1}}+2 a_{2} \frac{\partial L}{\partial z}+v_{2}\left(v_{1} \frac{\partial^{2} L}{\partial z \partial q_{1}}+a_{1} \frac{\partial^{2} L}{\partial z \partial v_{1}}+\left(v_{1} w_{2}-v_{2} w_{1}\right) \frac{\partial^{2} L}{\partial z^{2}}\right) \\
\Pi_{2}^{0}=\left.\frac{\partial L}{\partial v_{2}}\right|_{v_{1}, a_{1,2}}-\left.\frac{d}{d t} \frac{\partial L}{\partial a_{2}}\right|_{v_{1,2}, a_{1}}, \\
\Pi_{1}^{1}=\left.\frac{\partial L}{\partial a_{1}}\right|_{v_{1,2}, a_{2}}, \quad \Pi_{1}^{2}=\left.\frac{\partial L}{\partial a_{2}}\right|_{v_{1,2}, a_{1}}
\end{gathered}
$$


Reparametrization invariance is always, not only in this example, represented as homogeneity condition for a function, which depends on peculiar combinations

$$
z_{i j}=\dot{q}_{i} \ddot{q}_{j}-\ddot{q}_{i} \dot{q}_{j} \sim \partial_{t}\left(\frac{\dot{q}_{i}}{\dot{q}_{j}}\right), \quad z_{i j ; k l} \sim \partial_{t}\left(\frac{\dot{z}_{i j}}{\dot{z}_{k l}}\right), \quad z_{i j ; k l \mid i^{\prime} j^{\prime} ; k^{\prime} l^{\prime}} \sim \partial_{t}\left(\frac{\dot{z}_{i j ; k l}}{{\dot{z_{i}}}_{i^{\prime} ; k^{\prime} l^{\prime}}}\right), \ldots
$$

which are "elementary monomials", transforming homogeneously under the time-reparametrizations. Of course, they play essential role in the theory, see, for example, [40].

\section{Acknowledgements}

I am indebted for numerous discussion to Valery Dolotin and to other participants of his seminars at ITEP.

I acknowledge the hospitality of OCU and support of JSPS during completion of this text. This work is partly supported by Russian Federal Nuclear Energy Agency, by the joint grant 06-01-92059-CE, by NWO project 047.011.2004.026, by INTAS grant 05-1000008-7865, by ANR-05-BLAN-0029-01 project and by the Russian President's Grant of Support for the Scientific Schools NSh-8004.2006.2 and by RFBR grant 07-0200645 .

\section{References}

[1] E.Fradkin and A.Tseytlin, Quantum String Theory Effective Action, Nucl.Phys. B261 (1985) 1-27;

E.Witten, Noncommutative Geometry and String Field Theory, Nucl.Phys.B268 (1986) 253;

W.Siegel, Introduction to String Field Theory, World Scientific, 1988

[2] M.Reuter, Functional Renormalization Group Equations, Asymptotic Safety and Quantum Einstein Gravity, arXiv:0708.1317

[3] A.G.Lisi, An Exeptionally Simple Theory of Everything, arXiv:0711.0770

[4] N.Arkani-Hamed, S.Dimopoulos and G.Dvali, The hierarchy problem and new dimensions at a millimeter, Phys.Lett.B429 (1998) 263-272, hep-ph/9803315(1998); Large extra dimensions: A new arena for particle physics, Phys.Today 55N2 (2002) 35-40;

G.Landsberg, Collider Searches for Extra Dimensions, hep-ex/0412028, 2004;

A.Mironov, A.Morozov and T.Tomaras, Can Centauros or Chirons be the first observations of evaporating mini black holes?, hep-ph/0311318; If LHC is a mini-time-machines factory, can we notice?, Facta Univ.Ser.Phys.Chem.Tech.4 (2006) 381-404, arXiv:0710.3395;

I.Ya. Aref'eva, I.V. Volovich, Time Machine at the LHC, arXiv:0710.2696

[5] B.Kosyakov, Introduction to the Classical Theory of Particles and Fields, Springer, 2007;

D.Galtsov, Radiation Reaction in Various Dimensions, Phys.Rev. D66 (2002) 025016, hep-th/0112110;

P.Kazinsky, S.Lykhovich and A.Sharapov, Radiation Reaction and Renormalization in Classical Electrodynamics of a Point Particle in any Dimension, Phys.Rev. D66 (2002) 025017, hep-th/0201046;

Yu.Yaremko, Radiation Reaction, Renormalization and Poincare Symmetry, math-ph/0511075;

P.Kazinsky, Effective Dynamics of Singular Sources in Classical Field Theory, PhD thesis, Tomsk, 2007;

A.Mironov and A.Morozov, On the Problem of Radiation Friction Beyond 4 and 6 Dimensions, arXiv:0710.5676; hep-ph/0612074; hep-th/0703097;

D.Galakhov, Self-interaction and regularization of classical electrodynamics in higher dimensions, arXiv:0710.5688

[6] A.Slavnov, Renormalization of Supersymmetric Gauge Theories, Nucl.Phys.B97 (1975) 155;

for the most recent application of higher-derivative regularization see:

A.B.Pimenov, E.S.Shevtsova, A.A.Soloshenko and K.V.Stepanyantz, Higher derivative regularization and quantum corrections in $N=1$ supersymmetric theories, arXiv:0712.1721

[7] R.Woodard, A Canonical Formalism For Lagrangians With Nonlocality Of Finite Extent, Phys.Rev. A62 (2000) 05215, hep-th/0006207

[8] R.Miron, D.Hrimiue, H.Shimada and S.V.Sabau, The Geometry of Hamilton and Lagrange Spaces, Kluwer Academic Publishers, 2001 
[9] S.Hawking and T.Hertog, Living with Ghosts, Phys.Rev. D65 (2002) 103515, hep-th/0107088

[10] T.-C.Cheng, P.-M.Ho and M.-C.Yeh, Perturbative approach to higher derivative theories with fermions, Phys.Rev. D66 (2002) 085015, hep-th/0206077

[11] J.Gomis, K.Kamimura and T.Ramirez, Physical degrees of freedom of non-local theories, Nucl.Phys.B696 (2004) 263-291, hep-th/0311184

[12] A.Smilga, Ghost-free higher-derivative theory, Phys.Lett. B632 (2006) 433-438, hep-th/0503213; 6D Superconformal Theory as the Theory of Everything, hep-th/0509022

[13] M.Plyushchay, Majorana equation and exotics: Higher derivative models, anyons and noncommutative geometry, EJTP 3, \#10 (2006) 17-31, math-ph/0604022

[14] I.Cortese and J.A.Garcia, Equations of Motion, Noncommutativity and Quantization, Phys.Lett. A358 (2006) 327-333, hep-th/0605156; Int.J.Geom.Meth.Mod.Phys. 4 (2007) 789, hep-th/0703205

[15] S.Lyakhovich and A.Sharapov, Quantizing non-Lagrangian Gauge Theories: An Augmentation Method, JHEP 0701 (2007) 047, hep-th/0612086

[16] K.Bolonek and P.Kosinski On the unitarity of higher-dervative and nonlocal theories, J.Phys.A40 (2007) 11561-11568, quant-ph/0612091

[17] C.S.Acatrinei, A Path Integral Leading to Higher-Order Lagrangians, J.Phys.A40 (2007) F929-F933, arXiv:0708.4351

[18] K. Andrzejewski, J. Gonera and P. Maslanka, A Note on the Hamiltonian formalism for higher-derivative theories, arXiv:0710.2976

[19] M.Ostrogradski, Mem.Acad.St.Petersburg, VI 4 (1850) 385

[20] E.Wittaker, Analytic Dynamics, Cambridge University Press, 1937

[21] A.Pais and G.Uhlenbeck, Phys.Rev. 79 (1950) 145

[22] P.A.M.Dirac, Lectures on Quantum Mechanics, 1964

[23] L.Faddeev and A.Slavnov, Gauge Fields, Introduction to Quantum Theory, Benjamin, Reading, 1990

[24] B.A.Dubrovin, A.T.Fomenko and S.P.Novikov, Modern Geometry: Methods and Applications. Part II, The Geometry and Topology of Manifolds, Chapter 7, section 30, Springer (Graduate texts in Mathematics, 93), 1991

[25] D.M.Gitman and I.V.Tyutin, Quantization of fields with constraints, Springer-Verlag, 1990

[26] D.Eliezer and R.Woodard, The Problem of Nonlocality in String Theory, Nucl.Phys.B325 (1989) 389

[27] M.Plyushchay, Massless Point Particles with Rigidity, Mod.Phys.Lett. A4 (1989) 837-847

[28] J.M.Pons, Ostrogradski Theorem For Higher Order Singular Lagrangians, Lett.Math.Phys. 17 (1989) 181

[29] J.Z.Simon, Phys.Rev. D41 (1990) 3720

[30] M.Henneaux abd C.Teitelboim, Quantization of Gauge Systems, Princeton University Press, 1992

[31] J.Govaerts and M.S.Rashid, The Hamiltonian formulation of higher order dynamical systems, hepth/9403009

[32] J. Llosa and J. Vives, Hamiltonian formalism for nonlocal Lagrangians, J.Math.Phys. 35 (1994) 2856

[33] H.J.Schmidt, Stability and Hamiltonian formulation of higher derivative theories, Phys.Rev. D12 (1994) 6354 , gr-qc/9404038

[34] T.Nakamura and S.Hamamoto, Higher derivatives and canonical formalisms, Prog.Theor.Phys., 95 (1996) 409, hep-th/9511219 
[35] A.Morozov, Anomalies In Gauge Theories, Sov.Phys.Usp.29 (1986) 993-1039 [Usp.Fiz.Nauk 150 (1986) $337-416$;

A.Gorodentsev and A.Losev, talks at Dombay and Dubna Schools, 2004;

D.Krotov and A.Losev, Quantum field theory as effective BV theory from Chern-Simons, hep-th/0603201; P.Mnev, Notes on simplicial BF theory, hep-th/0610326

[36] V.Dolotin, A.Morozov and Sh.Shakirov, Higher Nilpotent Analogues of A-infinity Structure, Phys.Lett. B651 (2007) 71-73, arXiv:0704.2884; arXiv:0704.2609

[37] I.Gelfand, M.Kapranov and A.Zelevinsky, Discriminants, Resultants and Multidimensional Determinants, 1994, Birkhauser;

V.Dolotin and A.Morozov, Introduction to Non-Linear Algebra, World Scientific, 2007, hep-th/0609022

[38] D.Fairlie, J.Govaerts and A.Morozov, Universal Field Equations with Covariant Solutions, Nucl.Phys. B373 (1992) 214-232, hep-th/9110022

[39] V.Dolotin and A.Stoyanovsky, talks at ITEP seminars

[40] P.Dunin-Barkovsky and A.Sleptsov, Geometric Hamiltonian Formalism For Reparametrization Invariant Theories With Higher Derivatives, preprint ITEP/TH-59/07

[41] L.D.Landau and E.M.Lifshitz, Mechanics, 1976, Elsevier

[42] A.Morozov, Integrability and Matrix Models, Phys.Usp. 37 (1994) 1-55, hep-th/9303139; hep-th/9502091 